\title{
Dexmedetomidine protects against endothelial injury in septic rats induced by cecal ligation and puncture by decreasing angiopoietin 2 and increasing vascular endothelial cadherin levels
}

\author{
PENG ZHANG, JI PENG, YUN-QIN REN, HAN ZHENG and HONG YAN \\ Department of Anesthesiology, Daping Hospital, Army Medical University, Chongqing 400042, P.R. China
}

Received August 29, 2019; Accepted May 5, 2020

DOI: $10.3892 /$ etm.2020.9543

\begin{abstract}
The aim of the present study was to investigate the protective effect of dexmedetomidine (Dex) on endothelial injury in a cecal ligation and puncture (CLP)-induced rat model of sepsis. A total of 36 male Sprague-Dawley rats were divided into three groups: Sham, CLP and CLP + Dex. The wet/dry (W/D) ratio of lung weight, hematoxylin and eosin (H\&E) staining of lung tissue, plasma levels of angiopoietin (Ang)1 and 2, ratio of Ang2/1 and vascular endothelial (VE)-cadherin protein expression levels in lung tissue were determined. The W/D ratio of lung tissue in the CLP + Dex group was significantly lower than that in the CLP group $(\mathrm{P}<0.01)$. The H\&E staining results indicated that Dex treatment reduced the levels of CLP-induced alveolar septum widening, infiltrating white blood cells and congestion, when compared with CLP alone. In addition, the expression levels of plasma Ang2 and the Ang2/1 ratio in the CLP + Dex group were significantly lower than those of the CLP rats $(\mathrm{P}<0.01)$. Furthermore, the level of VE-cadherin protein in lung tissue of the CLP + Dex group was higher than that of the CLP group $(\mathrm{P}<0.05)$. The results indicated that Dex had a protective effect against CLP-induced endothelial injury, through the ability to reduce expression of the endothelial injury factor Ang2 and increase the expression of the endothelial adhesion junction factor VE-cadherin in a septic rat model. These data suggest a potential application of Dex in the clinical treatment of sepsis.
\end{abstract}

Correspondence to: Dr Hong Yan, Department of Anesthesiology, Daping Hospital, Army Medical University, 10 Changjiang Branch Road, Yuzhong, Chongqing 400042, P.R. China

E-mail: cqyanhong@163.com

Abbreviations: ang, angiopoietin; ARDS, acute respiratory distress syndrome; CLP, cecal ligation and puncture; Dex, dexmedetomidine; $\mathrm{H} \& \mathrm{E}$, hematoxylin and eosin; VE-cadherin, vascular endothelial cadherin; W/D, wet/dry

Key words: dexmedetomidine, angiopoietin-1, angiopoietin-2, sepsis, vascular endothelial cadherin, endothelial injury

\section{Introduction}

Sepsis is a systemic inflammatory response syndrome caused by bacterial infection and a life-threatening organ dysfunction syndrome. Globally, there are more than 18 million cases of sepsis every year. Despite considerable progress in anti-infection therapy and organ function support technology, the mortality rate of sepsis remains as high as $30-70 \%$. Approximately 14,000 individuals die from complications of sepsis worldwide every day and it is estimated that 215,000 individuals die in the United States every year (1). Statistical data from the United States indicate that the mortality rate of sepsis has exceeded that of ST-segment elevation myocardial infarction and has become the main cause of death in noncardiac patients in hospital intensive care units (2).

The underlying mechanism of sepsis is believed to involve complex systemic inflammatory network effects, gene polymorphisms, immune dysfunction, abnormal coagulation function, tissue damage and abnormal reactions of the host to pathogenic microorganisms and toxins from various infections. Under homeostatic conditions, the vascular endothelium can balance the levels of inflammatory mediators, the innate immune system and the coagulation system to coordinate the response to inflammation in the process of systemic inflammation, such as septic shock (3). However, once endothelial dysfunction is induced by sepsis, homeostasis imbalance, vascular reactivity changes and tissue edema may occur (4). Radeva and Waschke (5) demonstrated that inflammatory mediators could inhibit the signal transduction of the cAMP/Ras-related C3 botulinum toxin substrate 1 pathway and activate the Ras homolog family member A signaling pathway, leading to endocytosis, phosphorylation of endothelial adhesion junction proteins and depolymerization of tight junctions, eventually causing the destruction of the endothelial barrier. Moreover, Millar et al (6) established that the pulmonary vascular endothelium is a key regulator and coordinator of acute respiratory distress syndrome (ARDS). While describing the pathophysiological changes of the endothelium in patients with ARDS in detail, two potential signaling pathways for endothelial targeted therapy were explored: i) The sphingosine-1-phosphate pathway; and ii) the angiopoietin (Ang)-Ang1 receptor protein (Tie2) pathway (3). To the best 
of our knowledge, significant progress in the development of targeted drug therapy against these signaling pathways has notbeen made. Therefore, exploring new therapeutic methods is the focus of current research.

Dexmedetomidine (Dex) is an agonist of the adrenergic $\alpha_{2}$ receptor. Clinically, Dex can decrease sympathetic tone; therefore, it is used as a sedative and widely applied to inhibit the release of norepinephrine, thus terminating the propagation of pain signals (7). In addition to providing a good sedative effect, preclinical and clinical studies suggest that Dex has anti-inflammatory, antioxidative and stress control effects (8-11). However, no study has focused on the effects of Dex on endothelial damage in sepsis. In the present study, the protective effects of Dex on vascular endothelial injury in a cecal ligation and puncture (CLP)-induced rat model of sepsis were explored through detection of the expression levels of endothelial injury markers.

\section{Materials and methods}

Animals and group classification. A total of 36 male Sprague-Dawley rats aged at 6 weeks, weighing 200-220 g, were purchased from the Animal Laboratory of Daping Hospital, Army Medical University. All the procedures in the present study followed the principles of the International Guidelines on Animal Welfare (12). The animal experiments were approved by the Ethics Committee of Daping Hospital, Army Medical University (Chongqing, China).

The rats were randomly divided into three groups (12 rats/group): the Sham group, the CLP group and the CLP plus Dex intervention group (CLP + Dex group). Animals were caged with free access to food and water and were maintained under a 12 -h light/dark cycle at a temperature of $22 \pm 1^{\circ} \mathrm{C}$ and a humidity of $45 \%$. All experimental rats were anesthetized by intraperitoneal injection of $400 \mathrm{mg} / \mathrm{kg}(0.8 \mathrm{ml}$ of $10 \%)$ chloral hydrate (Shanghai Biochempartner) for CLP, sham operation, exsanguination or sample collection. Following chloral hydrate administration, no signs of peritonitis, pain or discomfort were found.

Rat CLP model. The animal model of sepsis in rats was set up according to the methods described by Rittirsch et al (13). Briefly, after intraperitoneal injection of $400 \mathrm{mg} / \mathrm{kg}(0.8 \mathrm{ml}$ of $10 \%$ ) chloral hydrate the rats were pinned on an operating table and the skin of the mid-lower abdomen of the rats was disinfected and incised. The cecum was found to the lower-left of the incision, squeezed to the distal part of the cecum and ligated at the distal end of the ileocecal base. The length of the ligation was $50 \%$ of the total length of the cecum. The ligated cecum was punctured with a needle on the side with less vasculature between the ligation line and the midpoint of the end of the cecum, and then some feces were squeezed out to kept the puncture hole passable, as previously described (14). The incision was finally closed and rinsed with saline to prevent rat dehydration. The CLP model was deemed to be accomplished with the manifesting signs of sepsis, including hypothermia, tachycardia, tachypnea, absence of grooming activities with resulting ruffled fur, reduced intake of food and water, lethargic conditions, elevated wet/dry (W/D) ratio of lung tissue and histological alterations. According to the

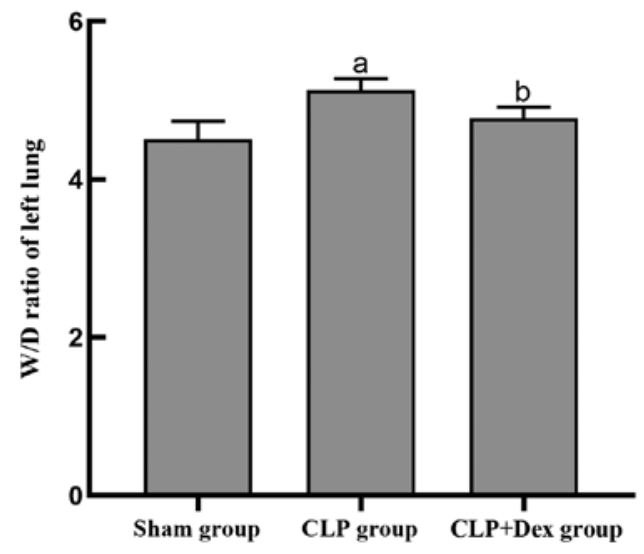

Figure 1. W/D weight ratio of lung tissue. The W/D ratios of the sham, CLP and Dex + CLP groups were measured. Each experiment was repeated six times. ${ }^{\text {a }} \mathrm{P}<0.05$, compared with the sham group. ${ }^{\mathrm{b}} \mathrm{P}<0.05$, compared with the CLP group. CLP, cecal ligation and puncture; Dex, dexmedetomidine; W/D, wet/dry.

methods described by Chen et al (15) and Hu et al (9), the rats in the CLP + Dex group were intraperitoneally injected with Dex $(10 \mu \mathrm{g} / \mathrm{kg}$; Jiangsu Hengrui Pharmaceutical Co., Ltd.) $30 \mathrm{~min}$ prior to the operation. Another dose of Dex $(10 \mu \mathrm{g} / \mathrm{kg})$ was administered $6 \mathrm{~h}$ after the operation. Meanwhile, the rats in the sham and the CLP groups were intraperitoneally injected with the equivalent volume of normal saline. In the sham group, the abdominal cavity of the rats was opened, the cecum kept outside of the abdominal cavity for $10 \mathrm{~min}$ and then returned into the abdominal cavity, before the incision was sutured layer by layer. The rats were then returned to their cages and provided with water and standard chow ad libitum. All rats were observed every $5 \mathrm{~min}$ for $12 \mathrm{~h}$ after the operation. Euthanasia was implemented according to the following symptoms indicating that the rats were moribund (16): Ruffled fur and piloerection, few or no activities when provoked, no response to touch stimuli, labored breathing with gasps and mostly or completely closed eyes when provoked. The surviving rats were injected intraperitoneally with $400 \mathrm{mg} / \mathrm{kg}$ chloral hydrate $(0.8 \mathrm{ml}$ of $10 \%)$ at $12 \mathrm{~h}$ after the operation. Blood samples $(2.5 \mathrm{ml} / \mathrm{rat})$ were collected from the abdominal aorta and the rats were sacrificed by exsanguination, before the lung samples were harvested for further analyses.

Plasma Ang1 and 2 detection with ELISA kits. Blood samples $(2.5 \mathrm{ml} / \mathrm{rat})$ were collected from the rat abdominal aorta. Plasma samples were obtained by collection of the supernatant from fresh blood after centrifugation at $7,000 \mathrm{x}$ g and $4^{\circ} \mathrm{C}$ for $10 \mathrm{~min}$. The samples were frozen immediately at $-80^{\circ} \mathrm{C}$. The Ang1 and 2 levels in the thawed plasma samples were detected by a Rat Ang1 ELISA kit (cat. no. EK1295) and a Rat Ang2 ELISA kit (cat. no. EK1574) (Wuhan Boster Biological Technology, Ltd.).

Hematoxylin and eosin $(H \& E)$ staining. The upper lobe of the right lung was fixed in $4 \%$ paraformaldehyde at $4^{\circ} \mathrm{C}$ for $24 \mathrm{~h}$ and dehydrated stepwise with gradient alcohol concentrations, washed with xylene, and embedded in paraffin. The lung tissue was sectioned at a $5-\mu \mathrm{m}$ thickness and stained with $\mathrm{H} \& \mathrm{E}$ as previously described (17). 

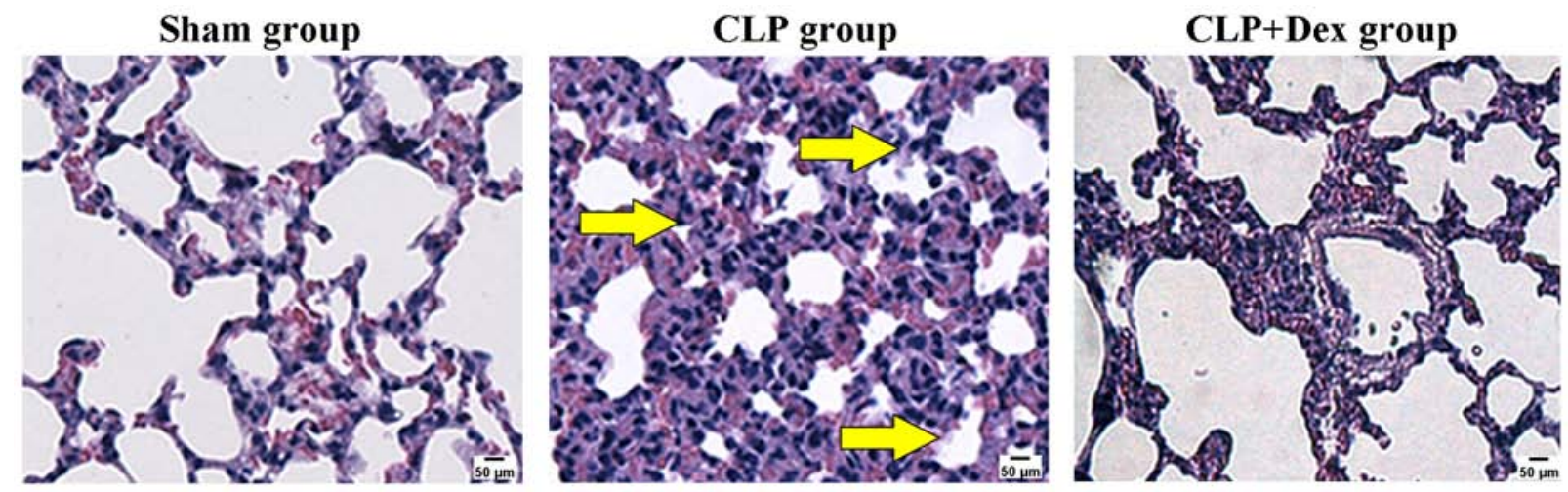

Figure 2. Dex significantly reduces the CLP-induced pulmonary edema as determined by H\&E staining of rat lung tissue. Representative images showing the H\&E staining of the upper lobe of right lung tissues of the rats in the sham, CLP and Dex + CLP groups. Arrows indicate the widened alveolar septum, infiltrated white blood cells, exuded edema fluid, as well as congestion in the CLP-treated rats compared with the sham rats. These alterations were markedly decreased in the Dex + CLP samples. CLP, cecal ligation and puncture; Dex, dexmedetomidine; H\&E, hematoxylin and eosin.

W/D weight ratio of lung tissue. Rats were sacrificed $12 \mathrm{~h}$ after the operation and the weight of the left lung tissue (wet weight) was measured by an electronic balance. Subsequently, the lung tissue was dried in an oven at $80^{\circ} \mathrm{C}$ for $48 \mathrm{~h}$ and the dry weight was measured. The W/D weight ratio was calculated as an index to indicate the degree of pulmonary edema.

Western blot analysis. The middle lobe of the rat right lung was immediately frozen in liquid nitrogen. Lung samples $(\sim 100 \mathrm{mg})$ were then homogenized in radioimmunoprecipitation assay buffer (Beyotime Institute of Biotechnology) containing protease inhibitors. Protein concentrations were determined using BCA assay kits (Thermo Fisher Scientific, Inc.; cat. no. 23227). For western blot analysis, samples containing $25 \mu \mathrm{g}$ of protein were loaded onto a $10 \%$ sodium dodecyl sulfate-polyacrylamide gel, electrophoresed, transferred onto a polyvinylidene difluoride membrane, blocked at room temperature for $60 \mathrm{~min}$ in 5\% skim milk and treated with primary antibodies against VE-cadherin (1:1,000 dilution) or GAPDH (1:1,000 dilution) as a protein loading reference (Thermo Fisher Scientific, Inc.; cat. nos. 36-1900 and MA5-15738-BTIN, respectively) at room temperature for $2 \mathrm{~h}$. After washing three times with Tris-buffered saline containing Tween-20 (TBST), the membrane was incubated with horseradish peroxidase-labeled secondary antibody (Thermo Fisher Scientific, Inc.; cat. no. A27036; 1:5,000 dilution) at room temperature for $1 \mathrm{~h}$, and washed again three times with TBST. Finally, the probed protein was visualized using enhanced chemiluminescence staining (BeyoECL Plus; Beyotime Institute of Biotechnology) on an Odyssey ${ }^{\circledR}$ CLx Infrared Imaging System (LI-COR Biosciences). The results were analyzed by densitometric measurement (ImageJ version 1.53a; National Institutes of Health) with a gel imager and the relative expression of VE-cadherin was normalized to GAPDH.

Statistical analysis. The experimental results were analyzed by using SPSS software (version 24; IBM Corp.). Data are presented as the mean \pm standard deviation. One-way analysis of variance was used for multiple-group comparisons followed by the Least Significant Difference post hoc test. $\mathrm{P}<0.05$ was considered to indicate a statistically significant difference.

\section{Results}

Dex relieves $C L P$-induced symptoms. Among the model rats, six from the CLP group and three from the CLP + Dex group reached the humane endpoints of the study. These rats were anesthetized by intraperitoneal injection of $400 \mathrm{mg} / \mathrm{kg}(0.8 \mathrm{ml}$ of $10 \%$ ) chloral hydrate and sacrificed by exsanguination. All rats in the sham group behaved normally when provoked, while rats in the CLP + Dex group showed an improved appetite, slightly ruffled fur, more active responses to auditory or touch stimuli, fewer periods of labored breathing and wider opened eyes than those of the CLP group (data not shown).

Dex prevents CLP-induced pulmonary edema. The W/D lung weight ratio of lung tissue reflects the amount of lung edema (18). To evaluate the protective effect of Dex on lung tissue edema, the W/D ratio of the lung tissue in each group was determined. The results indicated that the W/D ratio in the CLP group was significantly higher than that in the sham group $(\mathrm{P}<0.05)$; however, Dex treatment significantly reduced the increased ratio due to CLP when compared with CLP alone ( $\mathrm{P}<0.05$; Fig. 1$)$.

To confirm the edema-protective activity of Dex histologically, the lung tissues of each group were stained with H\&E. At $12 \mathrm{~h}$ after CLP, a widened alveolar septum, a large number of infiltrating white blood cells and congestion were clearly observed in the lung of the CLP group when compared with the sham group. The infiltrating white blood cells, widened alveolar septum and edema fluid and congestion in the CLP + Dex group were less obvious than in the CLP group (Fig. 2). These results indicated that CLP significantly induced edema of the rat lung and that Dex protected against CLP-induced pulmonary edema.

Dex pretreatment diminishes the CLP-induced increase in the plasma Ang2 level and the Ang2/1 ratio. The plasma Ang1 and 2 levels of the rats in the different groups were investigated. At $12 \mathrm{~h}$ after CLP treatment, the plasma Ang1 level in the CLP group was significantly lower than that in the sham group $(\mathrm{P}<0.01)$; however, no significant difference was found between the CLP and the CLP + Dex groups $(\mathrm{P}=0.85)$. However, the CLP group exhibited significantly 

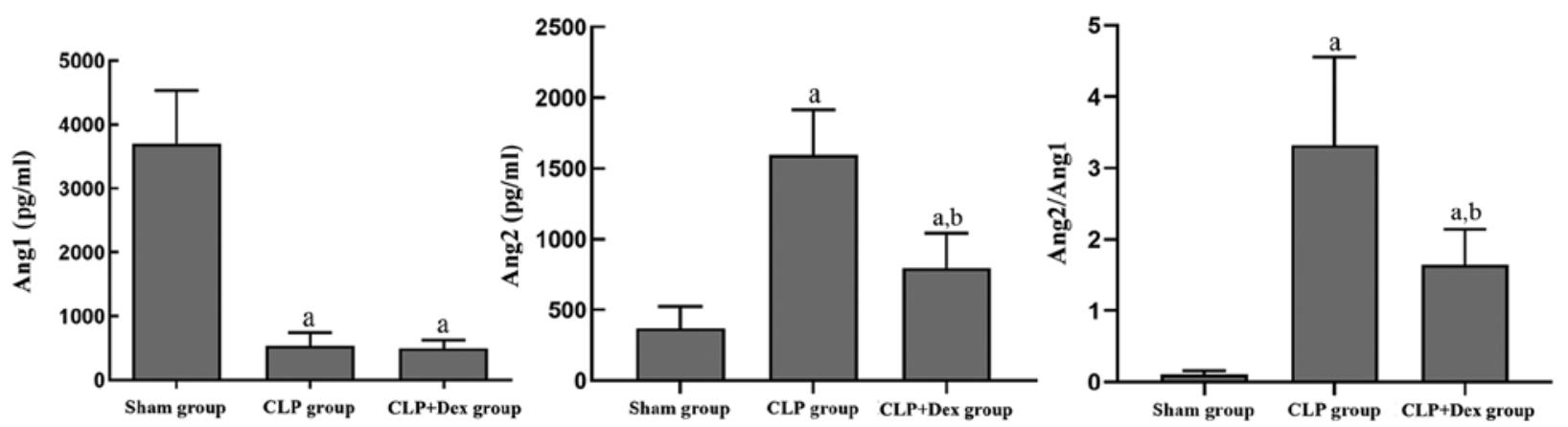

Figure 3. Dex significantly decreases the CLP-induced elevation of the plasma Ang2 level and the Ang2/1 ratio. The plasma levels of Ang1 and 2 were detected by the corresponding ELISA kits. The ratio of Ang2/1 was calculated based on the levels of Ang1 and 2. Each experiment was repeated six times. ${ }^{\mathrm{a}} \mathrm{P}<0.01$, compared with the sham group; ${ }^{\mathrm{b}} \mathrm{P}<0.01$, compared with the CLP group. Ang, angiopoietin; CLP, cecal ligation and puncture; Dex, dexmedetomidine.
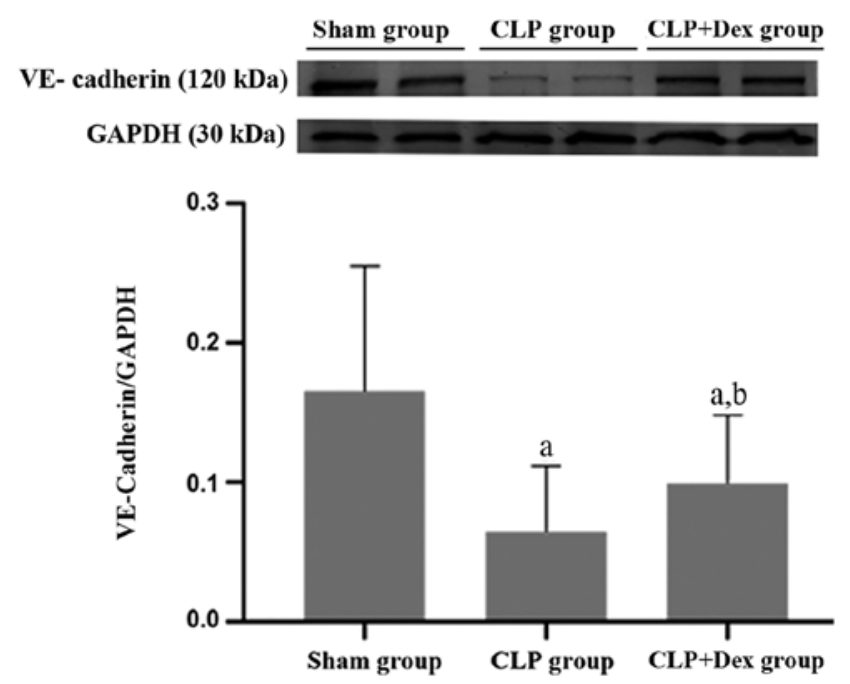

Figure 4. Dex alleviates the CLP-induced decrease in VE-cadherin protein expression. Protein samples of the rat middle lobe of right lung tissue from the sham, CLP and Dex + CLP groups were analyzed by western blotting with VE-cadherin antibody. GAPDH was used as a protein loading reference. The expression level of the protein was estimated by densitometry. The data are from three independent assays. ${ }^{\mathrm{a}} \mathrm{P}<0.05$, compared with the sham group. ${ }^{b} \mathrm{P}<0.05$, compared with the CLP group. CLP, cecal ligation and puncture; Dex, dexmedetomidine; VE-cadherin, vascular endothelial cadherin.

increased plasma Ang2 levels compared with those of the sham group $(\mathrm{P}<0.01)$. The increase in plasma Ang2 induced by CLP was reduced by pretreatment with Dex $(\mathrm{P}<0.01)$. Moreover, the ratio of Ang2/1 in the CLP group was significantly higher than that in the sham group $(\mathrm{P}<0.01)$ and that in the CLP + Dex group was lower than that in the CLP group $(\mathrm{P}<0.01)$. These data suggested that Dex may reverse the CLP-induced elevation of Ang2 expression and the Ang2/1 ratio (Fig. 3).

Dex alleviates the CLP-induced decrease in VE-cadherin protein expression in lung tissue. To investigate whether VE-cadherin is involved in CLP-induced sepsis, the protein level of VE-cadherin in the three groups was examined by western blot analysis. In the CLP + Dex group the protein expression of VE-cadherin in the lung tissue was significantly lower than that of the sham group $(\mathrm{P}<0.05)$. In addition, the level of VE-cadherin protein in the lung tissue of the
CLP + Dex group was significantly higher than that of the CLP group ( $\mathrm{P}<0.05$; Fig. 4$)$.

\section{Discussion}

To explore the complex molecular mechanism of sepsis, numerous animal models have been developed so far. Among these models, the most frequently applied one is the CLP model in rodents, which typically presents with clinical symptoms of sepsis and septic shock, including hypothermia, tachycardia and shortness of breath (13). Buras et al (19) reported that the CLP model is the 'gold standard' for laboratory sepsis research. In the present study, the advantages of the methods performed by Rittirsch et al (13) and Liu et al (14) were combined to establish a CLP model that successfully mimics typical symptoms of sepsis, such as hypothermia, tachycardia, tachypnea, absence of grooming activities with resulting ruffled fur, reduced intake of food and water and lethargic conditions. The sepsis model used in the current study was verified by the W/D lung weight ratio measurements and H\&E staining of lung tissue. The pathological alterations found in the present study were the same as those described by Li et al (10). This model provided a quick (12 h), simple, inexpensive and reproducible method for the study of sepsis.

During the process of sepsis, an excessive immune response involving the secretion of cytokines, chemokines and other activated factors has a pronounced impact on the whole body endothelium. The increased numbers of activated leukocytes adhere to the endothelium to trigger endothelial coagulation and destruction of the endothelial barrier. These alterations eventually lead to extensive global tissue edema (6). Compared with other tissues, there is a large amount of vasculature in lung tissue, providing abundant vascular endothelium (6). Therefore, the lung is severely affected by endothelial injury. Damage to the pulmonary vascular endothelial tissue leads to the accumulation of protein-rich fluid in the pulmonary interstitium, infiltration of the fluid into the alveolar cavity and eventually an imbalance of ventilation and blood flow (6). These pathological alterations further decrease the arterial oxygen partial pressure and pulmonary compliance, which are the common pathological alterations of ARDS (3). The present data supported these alterations and showed the signs of pulmonary edema, including an increased W/D lung weight ratio, widened pulmonary interstitium, and increased numbers 
of white and red blood cells, in the alveoli of CLP-induced septic rats.

Dex is a highly selective $\alpha 2$ receptor agonist that is widely used in the clinic. Its anti-inflammatory activity in preclinical and clinical experiments has attracted the interest of researchers (20). In addition to anti-inflammatory and antioxidative stress activities, Yeh et al (21) found that Dex could ameliorate the intestinal mucosa, seromuscular layer, aggregated lymphoid nodules and small vessel perfusion, while it reduced the endocan level and the destruction of tight junction protein in endotoxemic rats induced by lipopolysaccharide. Furthermore, the results of Miranda et al (22) suggested that Dex could reduce the rolling and adhesion of leukocytes to the endothelium in Syrian hamsters with endotoxemia as well as improve the condition of capillary perfusion. The results of the present study are consistent with the previous findings that pretreatment with Dex could significantly decrease the CLP-induced elevation of the W/D lung ratio. In addition, Dex treatment reduced the CLP-induced widening of the alveolar septum, infiltration of white blood cells, edema fluid exudation and congestion in sepsis model rats. All of the above results indicated that Dex may alleviate pulmonary edema through protection of endothelial barrier integrity.

The dose of Dex used in the present study was chosen according to previous studies $(9,23,24)$. A pilot study using Dex at a dose of $50 \mu \mathrm{g} / \mathrm{kg}$ was also conducted, but some rats showed significantly decreased respiratory frequencies and one of them manifested signs of hypoxia. Therefore, a dose of $10 \mu \mathrm{g} / \mathrm{kg}$, at which all experimental rats showed no obvious side effects, was chosen for the subsequent experiments. However, $10 \mu \mathrm{g} / \mathrm{kg}$ Dex surpasses the conventional dosage in clinical situations (7). The equivalent dose between rats and patients as well as the optimal dosage for critical patients should be further investigated.

Ang1 is synthesized and secreted by cells around the endothelium, while Ang2 is primarily produced by the endothelium and stored in Weibel-Palade bodies. Ang1 acts to stabilize endothelial adhesion through the Ang1-Tie2 signaling pathway, thus protecting the endothelium. Once inflammatory factors and other stimulatory molecules appear, Ang2 is rapidly released from Weibel-Palade bodies (25) to antagonize the anti-inflammatory effect of Ang1 by competing with the Tie2 receptor, leading to endothelial barrier destruction, activation of endothelial cells and promotion of vascular inflammation (26). The Ang-Tie2 axis is one of the most widely studied pathways in the field of endothelial barrier function regulation. Elevation of Ang2 levels in plasma indicates progressive injury of endothelial cells, which can predict mortality in sepsis-related ARDS patients (26). In sepsis, ARDS, and related cases, the circulating Ang1 level has been reported to be 2-3 times less than normal levels; in contrast, the plasma Ang2 levels are increased by 5-20 times (27). Therefore, the ratio of Ang2/1 can better reflect the severity of endothelial damage than either Ang1 or 2 alone. In addition, Han et al (28) found that the circulating Ang2 level reached a peak at $12 \mathrm{~h}$ after CLP stimulation. Therefore, stimulation with CLP for $12 \mathrm{~h}$ was carried out in order to investigate the effect of Dex on the expression of Ang2 and 1 as well as the Ang2/1 ratio in sepsis model rats in the present study. The results of the present study demonstrated that CLP stimulation significantly elevated the plasma Ang2 level and the Ang2/1 ratio in sepsis model rats. Dex successfully reduced the CLP-upregulated plasma Ang2 level and the Ang2/1 ratio in sepsis model rats.

VE-cadherin is a key molecule in the adhesion junctions between vascular endothelial cells (5). Ang1 can bind and activate the Tie 2 receptor to trigger downstream guanosine triphosphatase to release phosphate, which stabilizes the cellular barrier through upregulation of VE-cadherin in cellular junctions (5). In the present study, the VE-cadherin levels in the lung tissue of the rats pretreated with Dex were significantly greater compared with the CLP group, which may be caused by downregulation of Ang2 expression by Dex through the Ang-Tie2-VE-cadherin signaling pathway. Although Dex did not directly affect Ang1 expression compared with CLP alone, the Ang2/1 ratio in the CLP + Dex group was significantly reduced. The reduced ratio of Ang2/1 may reduce the phosphorylation and internalization of VE-cadherin through the Ang1-Tie2-VE-cadherin signaling pathway, ultimately reducing the damage of the endothelial barrier function and alleviating pulmonary edema.

In the present study, the plasma Ang2 level in the CLP group was not as high as reported previously by Ricciuto et al (27). The reason for this may be that rodents have a strong resistance to bacteria (19); therefore, the vascular endothelial damage by CLP is not as obvious as that in humans. Furthermore, species differences may be involved in the differences as well (29). Further determination of the suitable dosage of Dex in rats and reasonable estimation of the equivalent dosage among different species requires additional exploration. The results of the present study suggest that Dex reduced the Ang2/1 ratio by decreasing the Ang2 level, which may trigger Ang-Tie2 signaling to affect VE-cadherin expression and improve endothelial barrier function. Sepsis is a highly heterogeneous disorder with a complex pathogenesis, and endothelial injury in sepsis involves abnormalities of inflammation (30), immunity (31), complement, coagulation (32), the endothelial system and multiple signaling pathways (4). There remain numerous regulatory factors and signaling pathways upstream of VE-cadherin, which require further exploration to verify whether Dex can play a role in other stable signaling pathways of endothelial barrier function.

The present study explored the effects of Dex, a high-affinity $\alpha-2$ receptor agonist, on endothelial injury in a CLP-induced sepsis model. The results demonstrated that Dex attenuated the elevation of plasma Ang2 to reduce the ratio of Ang2/1, which may have consequently mitigated the decrease in the expression level of VE-cadherin. The present study had certain limitations. The reasons why Dex reduced the plasma Ang2 level but did not have an impact on the Ang1 level were not determined. Additionally, considering that other signaling pathways contribute to the maintenance of endothelial function, further investigations are warranted to explore the specific mechanisms.

The results of the present study suggested that Dex may decrease CLP-induced upregulation of Ang2 expression and increase the CLP-induced decrease of VE-cadherin expression in a sepsis rat model, and protect against pulmonary edema. These data provide molecular evidence to support the clinical application of Dex for the treatment of sepsis. 


\section{Acknowledgements}

Not applicable.

\section{Funding}

No funding was received.

\section{Availability of data and materials}

The datasets used and/or analyzed during the current study are available from the corresponding author on reasonable request.

\section{Authors' contributions}

PZ wrote the original draft, conceived and designed the experiments and performed the animal experiment. JP participated in the animal experiments and collected the data. YQR performed the data analysis and contributed to the manuscript preparation and data presentation. HZ contributed to the reagents, materials, data analysis and the guidance of experimental procedures. HY revised and edited the manuscript, as well as provided the general supervision of the research group. All authors read and approved the final manuscript.

\section{Ethics approval and consent to participate}

All the procedures of this study followed the principles of the International Guidelines on Animal Welfare. The animal experiments were approved by the Ethics Committee of Daping Hospital, Army Medical University.

\section{Patient consent for publication}

Not applicable.

\section{Competing interests}

The authors declare that they have no competing interests.

\section{References}

1. Cecconi M, Evans L, Levy M and Rhodes A: Sepsis and septic shock. Lancet 392: 75-87, 2018.

2. Singer M, Deutschman CS, Seymour CW, Shankar-Hari M, Annane D, Bauer M, Bellomo R, Bernard GR, Chiche JD, Coopersmith CM, et al: The third international consensus definitions for sepsis and septic shock (sepsis-3). JAMA 315 801-810, 2016

3. Gotts JE and Matthay MA: Sepsis: Pathophysiology and clinical management. BMJ 353: i1585, 2016.

4. Ince C, Mayeux PR, Nguyen T, Gomez H, Kellum JA, Ospina-Tascón GA, Hernandez G, Murray P and De Backer D ADQI XIV Workgroup: The endothelium in sepsis. Shock 45: 259-270, 2016.

5. Radeva MY and Waschke J: Mind the gap: Mechanisms regulating the endothelial barrier. Acta Physiol (Oxf): 222, 2018. doi: 10.1111/apha.12860.

6. Millar FR, Summers C, Griffiths MJ, Toshner MR and Proudfoot AG: The pulmonary endothelium in acute respiratory distress syndrome: Insights and therapeutic opportunities. Thorax 71: 462-473, 2016.

7. Weerink MAS, Struys MMRF, Hannivoort LN, Barends CRM, Absalom AR and Colin P: Clinical pharmacokinetics and pharmacodynamics of dexmedetomidine. Clin Pharmacokinet 56: 893-913, 2017.
8. Meng L, Li L, Lu S, Li K, Su Z, Wang Y, Fan X, Li X and Zhao G: The protective effect of dexmedetomidine on LPS-induced acute lung injury through the HMGB1-mediated TLR4/NF- $\kappa \mathrm{B}$ and PI3K/Akt/mTOR pathways. Mol Immunol 94: 7-17, 2018.

9. Hu H, Shi D, Hu C, Yuan X, Zhang J and Sun H: Dexmedetomidine mitigates CLP-stimulated acute lung injury via restraining the RAGE pathway. Am J Transl Res 9: 5245-5258, 2017.

10. Li B, Li Y, Tian S, Wang H, Wu H, Zhang A and Gao C: Anti-inflammatory effects of perioperative dexmedetomidine administered as an adjunct to general anesthesia: A meta-analysis. Sci Rep 5: 12342, 2015.

11. Wang XW, Cao JB, Lv BS, Mi WD, Wang ZQ, Zhang C, Wang HL and $\mathrm{Xu} Z$ : Effect of perioperative dexmedetomidine on the endocrine modulators of stress response: A meta-analysis. Clin Exp Pharmacol Physiol 42: 828-836, 2015.

12. International guidelines on animal welfare. 156: 723-723, 2005.

13. Rittirsch D, Huber-Lang MS, Flierl MA and Ward PA Immunodesign of experimental sepsis by cecal ligation and puncture. Nat Protoc 4: 31-36, 2009.

14. Liu X, Wang N, Wei G, Fan S, Lu Y, Zhu Y, Chen Q, Huang M, Zhou $\mathrm{H}$ and Zheng J: Consistency and pathophysiological characterization of a rat polymicrobial sepsis model via the improved cecal ligation and puncture surgery. Int Immunopharmacol 32: 66-75, 2016.

15. Chen Q, Yi B, Ma J, Ning J, Wu L, Ma D, Lu K and Gu J: $\alpha 22$-adrenoreceptor modulated FAK pathway induced by dexmedetomidine attenuates pulmonary microvascular hyper-permeability following kidney injury. Oncotarget 7 : 55990-56001, 2016.

16. Mai SHC, Sharma N, Kwong AC, Dwivedi DJ, Khan M, Grin PM, Fox-Robichaud AE and Liaw PC: Body temperature and mouse scoring systems as surrogate markers of death in cecal ligation and puncture sepsis. Intensive Care Med Exp 6: 20, 2018.

17. Cox RA: Endothelin-1 and acute lung injury in sheeps with smoke inhalation and burn injury[D]. Galveston: The University of Texas,Graduate School of Biomedical Sciences: 18-19, 2003.

18. Jin Y, Yu G, Peng P, Zhang Y and Xin X: Down-regulated expression of AQP5 on lung in rat DIC model induced by LPS and its effect on the development of pulmonary edema. Pulm Pharmacol Ther 26: 661-665, 2013.

19. Buras JA, Holzmann B and Sitkovsky M: Animal models of sepsis: Setting the stage. Nat Rev Drug Discov 4: 854-865, 2005.

20. Cruz FF, Rocco PR and Pelosi P: Anti-inflammatory properties of anesthetic agents. Crit Care 21: 67, 2017.

21. Yeh YC, Wu CY, Cheng YJ, Liu CM, Hsiao JK, Chan WS, Wu ZG, Yu LC and Sun WZ: Effects of Dexmedetomidine on intestinal microcirculation and intestinal epithelial barrier in endotoxemic rats. Anesthesiology 125: 355-367, 2016.

22. Miranda ML, Balarini MM and Bouskela E: Dexmedetomidine attenuates the microcirculatory derangements evoked by experimental sepsis. Anesthesiology 122: 619-630, 2015.

23. Wu Y, Liu Y, Huang H, Zhu Y, Zhang Y, Lu F, Zhou C, Huang L, $\mathrm{Li} \mathrm{X}$ and Zhou C: Dexmedetomidine inhibits inflammatory reaction in lung tissues of septic rats by suppressing TLR4/NF- $\kappa B$ pathway. Mediators Inflamm 2013: 562154, 2013.

24. Ma Y, Yu XY and Wang Y: Dose-related effects of dexmedetomidine on immunomodulation and mortality to septic shock in rats. World J Emerg Med 9: 56-63, 2018.

25. Moss A: The angiopoietin: Tie 2 interaction: A potential target for future therapies in human vascular disease. Cytokine Growth Factor Rev 24: 579-592, 2013.

26. Parikh SM: Dysregulation of the angiopoietin-Tie-2 axis in sepsis and ARDS. Virulence 4: 517-524, 2013.

27. Ricciuto DR, dos Santos CC, Hawkes M, Toltl LJ, Conroy AL, Rajwans N, Lafferty EI, Cook DJ, Fox-Robichaud A, Kahnamoui K, et al: Angiopoietin-1 and 2 as clinically informative prognostic biomarkers of morbidity and mortality in severe sepsis. Crit Care Med 39: 702-710, 2011.

28. Han S, Lee SJ, Kim KE, Lee HS, Oh N, Park I, Ko E, Oh SJ, Lee YS, Kim D, et al: Amelioration of sepsis by TIE2 activation-induced vascular protection. Sci Transl Med 8: 335ra355, 2016.

29. Poli-de-Figueiredo LF, Garrido AG, Nakagawa N and Sannomiya P: Experimental models of sepsis and their clinical relevance. Shock 30 (Suppl 1): S53-S59, 2008.

30. Chousterman BG, Swirski FK and Weber GF: Cytokine storm and sepsis disease pathogenesis. Semin Immunopathol 39: 517-528, 2017.

31. van der Poll T, van de Veerdonk FL, Scicluna BP and Netea MG: The immunopathology of sepsis and potential therapeutic targets. Nat Rev Immunol 17: 407-420, 2017.

32. Levi $\mathrm{M}$ and van der Poll T: Coagulation and sepsis. Thromb Res 149: 38-44, 2017.

This work is licensed under a Creative Commons Attribution-NonCommercial-NoDerivatives 4.0 International (CC BY-NC-ND 4.0) License. 